\title{
A Micromixer Using the Chaos of Secondary Flow: Rotation Effect of Channel on the Chaos of Secondary Flow
}

\author{
Yasutaka Hayamizu $^{1}$, Shinichiro Yanase ${ }^{2}$, Shinichi Morita ${ }^{1}$, Shigeru Ohtsuka ${ }^{1}$, \\ Takeshi Gonda ${ }^{1}$, Kazunori Nishida ${ }^{2}$, Kyoji Yamamoto ${ }^{2}$ \\ ${ }^{1}$ Department of Mechanical Engineering, Yonago National College of Technology, Tottori, Japan \\ ${ }^{2}$ Graduate School of Natural Science and Technology, Okayama University, Okayama, Japan \\ Email: hayamizu@yonago-k.ac.jp
}

Received September 3, 2012; revised October 11, 2012; accepted October 23, 2012

\begin{abstract}
The micromixer, which has a rotor with a curved channel, is studied experimentally. The secondary flow in a curved channel of rectangular cross-section is investigated using PIV (Particle Image Velocimetry) and LIF (Laser Induced Fluorescence) methods. Two walls of the channel (the inner and top walls) rotate around the center of curvature and a pressure gradient is imposed in the direction of the exit of the channel. The non-dimensional channel curvature $\delta=a / R$ is taken to be about 0.1 , where $2 a$ is the width of the channel, $R$ the curvature radius of the channel. Other non-dimensional parameters concerned are the Dean number $\operatorname{De}=\operatorname{Re} \delta^{1 / 2}$, the Reynolds number $\operatorname{Re}=q d_{h} / v$, where $q$ is the mean flow velocity in the channel axis direction, $v$ the kinematic viscosity, $d_{h}$ the hydraulic diameter of the channel, and the Taylor number $\operatorname{Tr}=2(2 \delta)^{1 / 2} \Omega a^{2} /(\delta v)$, where $\Omega$ is the angular velocity of the rotor. Photographs of the flow in a cross-section at $180^{\circ}$ downstream from the curved channel entrance are taken by changing the flux (De) at a constant rotational speed $(\mathrm{Tr})$ of the channel walls. It is found that good mixing performance is obtained in the case of $\mathrm{De} \leq 0.1|\operatorname{Tr}|$ and for that case secondary flows show chaotic behaviors. And then we have confirmed the occurrence of reversal of the mean axial flow.
\end{abstract}

Keywords: Chaos; Rotation Effect; Secondary Flow; PIV; LIF

\section{Introduction}

Recently, a great attention has been paid to the development of a micro-chemical-analysis device called $\mu$ TAS (Micro Total Analysis Systems) in the field of the chemistry and biotechnology. This device, which consists of various micro-flow devices and sensors, functions in a series of operation such as mixture, reaction, separation and extraction. However, the flow is in the low Reynolds number region because of the micro-size of the channel, so that we cannot expect mixing effect by turbulence. Therefore, other mechanism is needed to mingle low Reynolds number flows efficiently in the micromixer.

The micromixers of various methods have been studied so far. Since a micromixer with comparatively simple shape among them can be made using the method of creating secondary flows within the channel, the study of the micromixer of this type is important in a practical use of $\mu$ TAS. Stroock et al. [1] studied a micromixer generating secondary flows in a channel by carving a ditch on the channel wall surface. Kim et al. [2] also studied a micromixer using a similar method. Sato et al. [3] made a micromixer which generates stronger secondary flows by carving ditches on the three wall surfaces of the channel. It has been shown that these methods are effective when the flow velocity is fast, though the pressure loss becomes a serious problem in this case. On the other hand, the micromixer which uses the chaos of the flow was studied by Niu et al. [4] and Tabeling et al. [5]. It was shown that the flow is miscible within a relatively short channel distance compared with that by the mixing using only non-chaotic secondary flows. Therefore, we propose a micromixer making use of the chaos of the secondary flow, specifically one in which the secondary flow becomes chaotic through a curved channel where two walls of the channel rotate. 
In this paper, we produced a micromixer model of the curved channel several centimeters long with rectangular cross-section of a few millimeters side. The secondary flow was measured using PIV (Particle Image Velocimetry) and LIF (Laser Induced Fluorescence) methods to examine the secondary flow characteristics for the effects of rotation and aspect ratio of the channel.

\section{Experimental Method}

\subsection{Experimental Setup}

A diagram of the experimental setup is shown in Figure 1. Two working fluids ( $70 \mathrm{wt} \%$ of glycerol aqueous solution and $70 \mathrm{wt} \%$ of glycerol aqueous solution dissolving rhodamine $\mathrm{B}$ at the $2.5 \mathrm{ppm}$ concentration) which are reserved in the tank (1), (2) are drawn to the overflow tank (5), (6) by the pump (3), (4). Then, it flows into the curved channel, a test section (9), via the inlet tube (7) and goes to the drain tank. The test section consists of two parts, i.e., the rotor and the casing. The channel is formed of the casing and the rotor where the upper wall and right (inner) wall of the channel are the rotor walls capable of being rotated (refer to Figure 2) when one sees from the upstream of the entrance. The rotor is rotated by the motor (8). The flux of the test section can be controlled by the needle valve (10) which is at the exit of the test section. We measured the number of revolutions of the rotor and the weight of outflow working fluid during a certain time. A viewing block is installed to ease the refraction of light in case of visualization of the secondary flow. We used Davis 7.2 (LaVision) for PIV and LIF, and hollow-glass particles (particle diameter 9 - 13 $\mu \mathrm{m})$ of a specific gravity 1.1 for tracer of PIV.

\subsection{Curved Channel and Method of Visualization}

The dimension of the curved channel is shown in Table 1. Here, $2 a$ is the width of the curved channel, $2 b$ the height of the curved channel, $R$ the radius of curvature of the center line of the curved channel, and $l$ the total

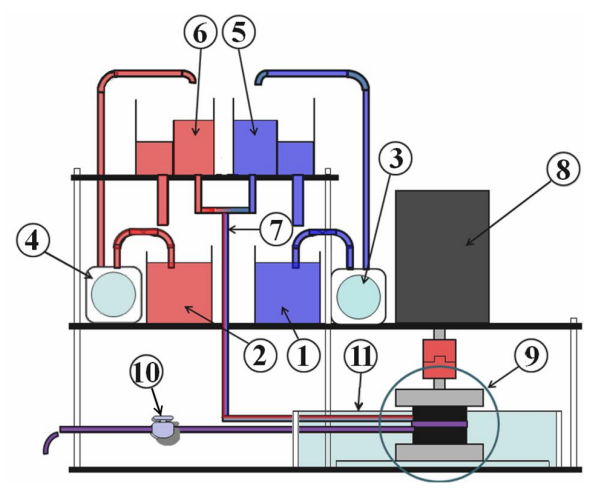

Figure 1. Diagram of the experimental setup.

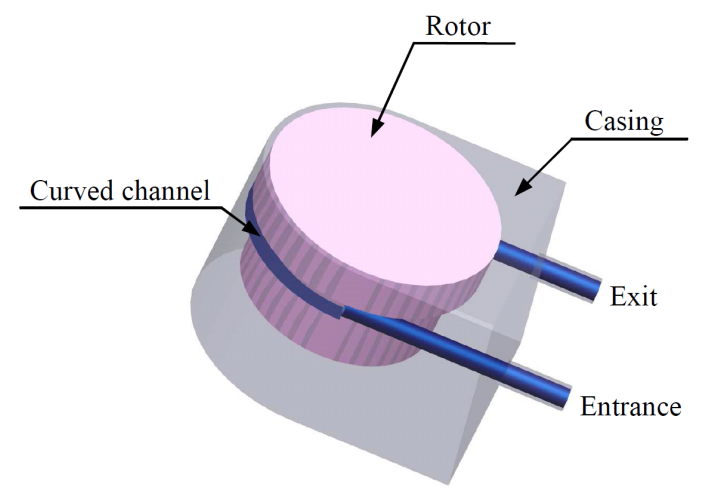

(a)

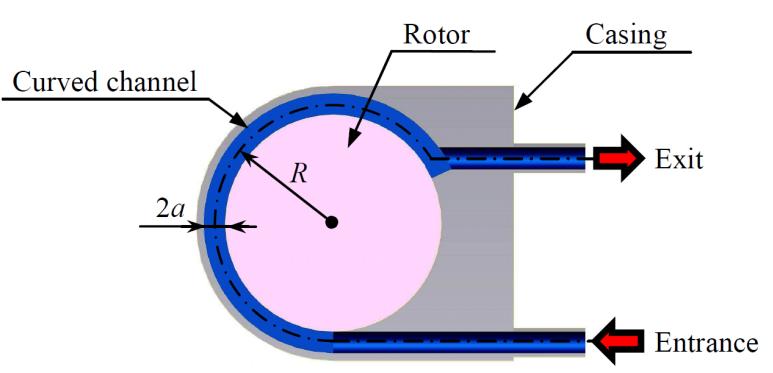

(b)

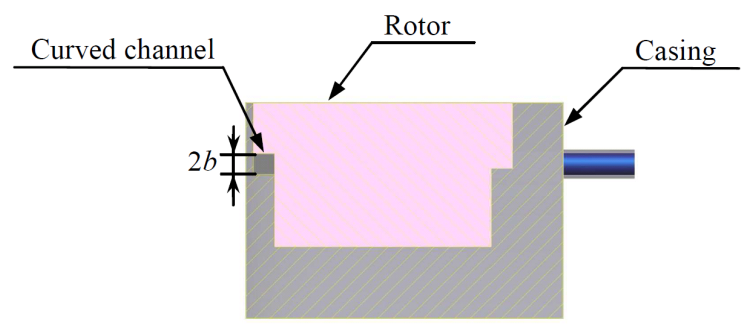

(c)

Figure 2. Enlargement of the test section (Mixer I). (a) 3-D view of the test section; (b) Top view of the test section; (c) Cross-section view of the test section.

Table 1. Dimensions of curved channel.

\begin{tabular}{ccccccc}
\hline Mixer & $2 a[\mathrm{~mm}]$ & $2 b[\mathrm{~mm}]$ & $R[\mathrm{~mm}]$ & $l[\mathrm{~mm}]$ & $\gamma$ & $\delta$ \\
\hline I & 3 & 3 & 15 & 58.9 & 1 & 0.1 \\
II & 4.5 & 2 & 22.5 & 88.4 & 0.44 & 0.1 \\
III & 2 & 4.5 & 10 & 39.3 & 2.25 & 0.1 \\
\hline
\end{tabular}

length of the channel. The aspect ratio $\gamma$ and the nondimensional curvature $\delta$ are given by

$$
\begin{aligned}
& \gamma=\frac{b}{a}, \\
& \delta=\frac{a}{R} .
\end{aligned}
$$

Other non-dimensional parameters concerned are the Reynolds number Re, the Dean number De [6] and the Taylor number $\operatorname{Tr}$ [7] given by 


$$
\begin{aligned}
& \operatorname{Re}=\frac{q d_{h}}{v}, \\
& \operatorname{De}=\operatorname{Re} \sqrt{\delta}, \\
& \operatorname{Tr}=\frac{2 a^{2} \Omega \sqrt{2 \delta}}{\delta v},
\end{aligned}
$$

where $q$ is the mean flow velocity in the channel axis direction, $d_{h}=4 a b /(a+b)$ the hydraulic diameter of the channel, $v$ the kinematic viscosity, and $\Omega$ the angular velocity of the rotor. If the rotation of the rotor is in the same direction as the mean flow in the channel, the parameter $\Omega$ is taken to be positive; otherwise, it is negative.

Next we explain the method of visualization of the flow. A scheme of the method of visualization is shown in Figure 3. The laser sheet lights up a cross-section of the channel normal to the channel side wall and the photographs of the secondary flow patterns are taken by a high speed camera.

We acquired the image of only the hollow-glass particles by installing the band-pass filter (center wave length of $532 \mathrm{~nm}$ and full width at half maximum of $10 \mathrm{~nm}$ ) in a high speed camera for PIV to calculate the velocity distribution. We acquired the fluorescence only of the rhodamine $\mathrm{B}$ by installing the high-pass filter (transmitted wave length of $570 \mathrm{~nm}$ or more) in a high speed camera for LIF to calculate the concentration distribution.

We also measured the viscosity of glycerol aqueous solution using the precision rotational viscometer before and after the experiment and confirmed that there was no viscosity change throughout the experiment. The density of glycerol aqueous solution was calculated by use of the gravimeter.

\section{Experimental Results and Discussion}

The experiment was carried out in the range of the Dean number from 0 to 1 . The Taylor number are taken to be 0 , 3 and -3. Figures 4-6, 10 and 11 show LIF and PIV re-

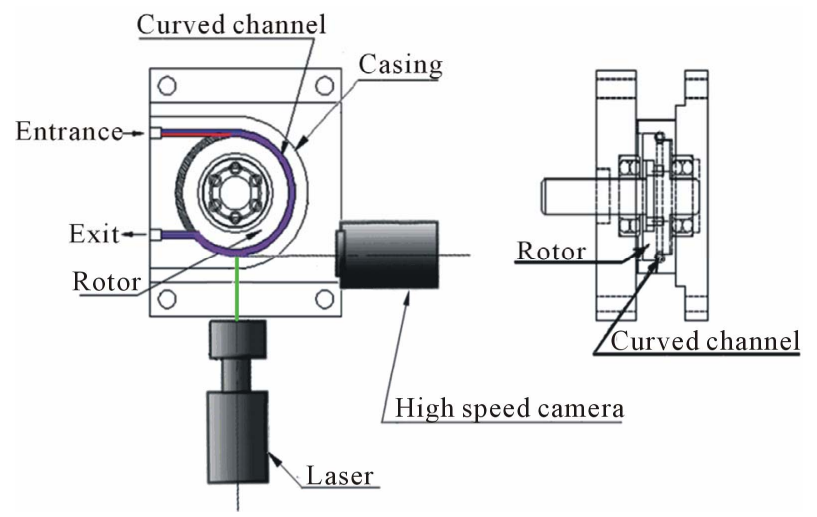

Figure 3. Scheme of the method of visualization.

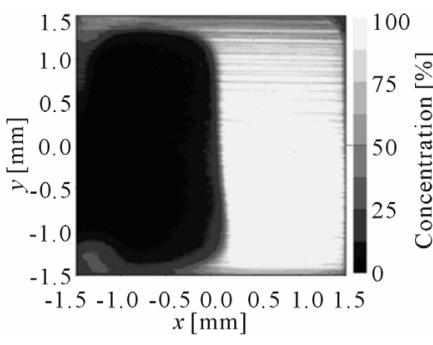

(i) LIF

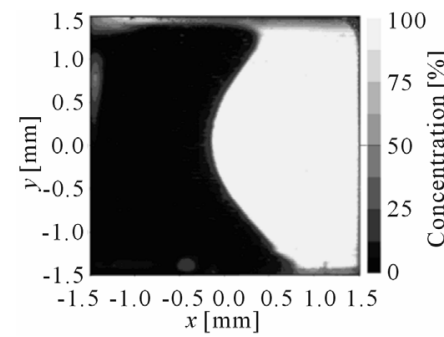

(i) LIF

(a)

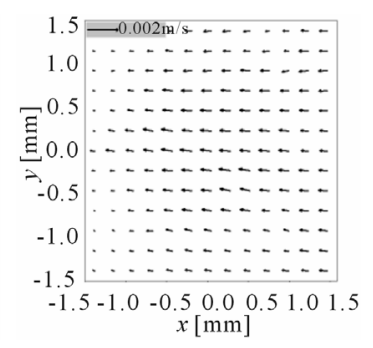

(ii) PIV

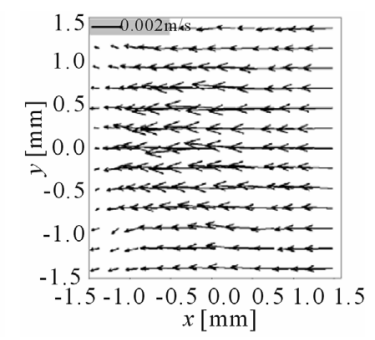

(ii) PIV

(b)

Figure 4. Secondary flow pattern of Mixer $I$ at $\mathbf{T r}=\mathbf{0}$. (a) $\mathrm{De}=0.3$; (b) De $=1$.

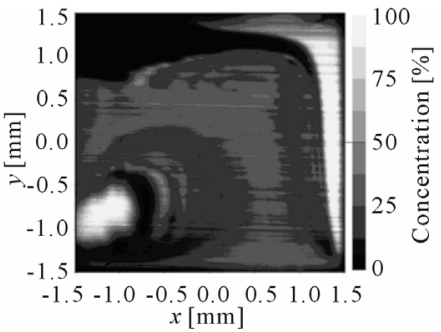

(i) LIF

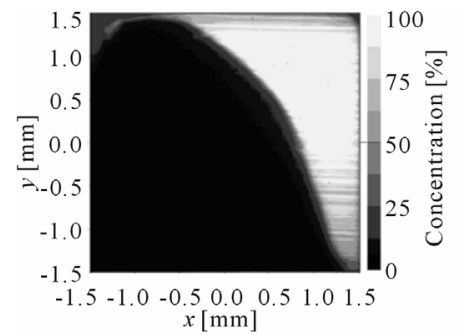

(i) LIF

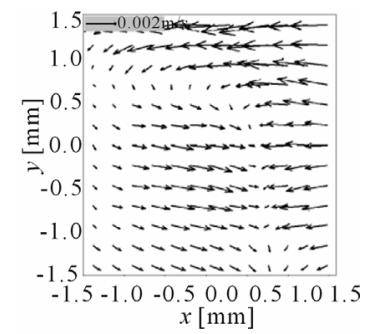

(ii) PIV

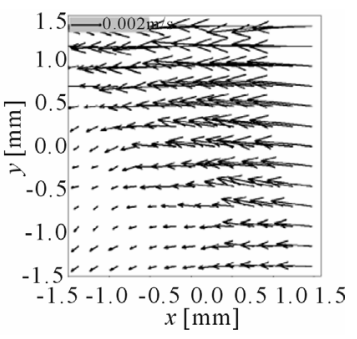

(ii) PIV (b)

Figure 5. Secondary flow pattern of Mixer $I$ at $\operatorname{Tr}=3$. (a) $\mathrm{De}=0.3$; (b) $\mathrm{De}=1$.

sults of the secondary flow. Figures in a cross-section at $180^{\circ}$ downstream from the curved channel entrance are seen from the upstream. The top and right of each figure are on a rotor wall. The value of the Dean number De is shown at the bottom of the figures. The bar display on the right-hand side of the LIF figures shows concentration of the glycerol aqueous solution including rhodamine B, 


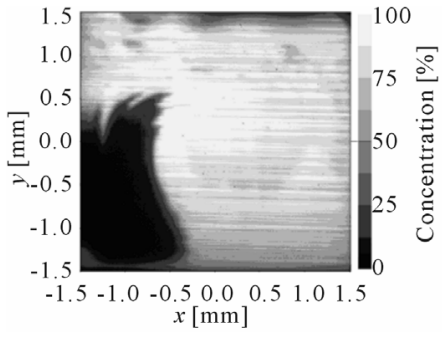

(i) LIF

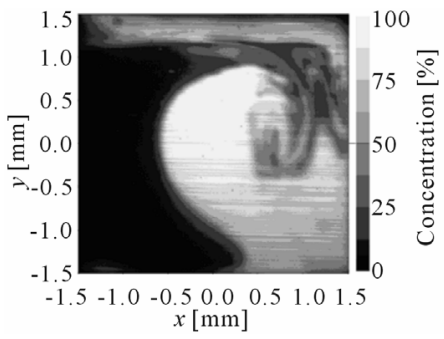

(i) LIF

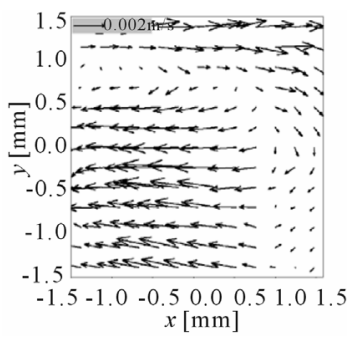

(ii) PIV

(a)

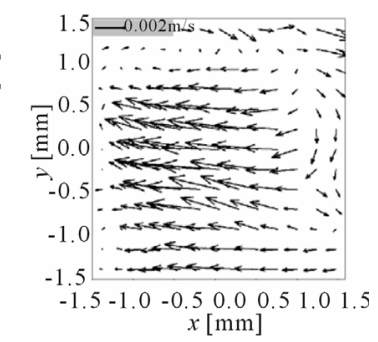

(ii) PIV (b)

Figure 6. Secondary flow pattern of Mixer $I$ at $\mathrm{Tr}=-3$. (a) $\mathrm{De}=0.3 ;$ (b) $\mathrm{De}=1$.

and the arrow in the upper left end of the PIV figures shows the flow velocity of $0.002 \mathrm{~m} / \mathrm{s}$. Two working fluids flow in two layers on the right and left sides, respectively, with the same mass flux where a glycerol aqueous solution including rhodamine $\mathrm{B}$ is on the right (rotor) side.

\subsection{Mixer I}

Figure 4(a) is the case for $\operatorname{Tr}=0$ and $\operatorname{De}=0.3$. The result of LIF shows that two fluid layers are separated similarly as the entrance flow. As for the secondary flow, the result of PIV shows that the flow velocity is faster in the central region of the cross-section, and the flow is steady, wholly directed to the same direction (to the casing side). Figure 4(b) is the case for $\operatorname{Tr}=0$ and $\mathrm{De}=1$. The mixing of two fluids does not occur though the result of LIF shows that the centre part of the boundary of two fluids is pulled to the casing side because of the centrifugal force by the curved channel. The result of PIV shows that the secondary flow velocity increases compared with the case of $\mathrm{De}=0.3$.

In the case for $\mathrm{Tr}=3$ and $\mathrm{De}=0.3$, there exists very small area of concentration of $0 \%$ (glycerol aqueous solution) or $100 \%$ (glycerol aqueous solution including rhodamine B) where two fluids are not mixed, and good mixing is achieved by the rotation effect of the channel as shown by the result of LIF in Figure 5(a). In Figure 6(a), the LIF result for $\operatorname{Tr}=-3$ and $\mathrm{De}=0.3$ is displayed. It is found that the area where two fluids are not mixed reduces. The PIV results show that the secondary flow pattern is greatly different from the case for $\operatorname{Tr}=0$ due to the rotation effect of the channel. It is noteworthy that the secondary flow becomes unsteady. Good mixing was achieved for De $\leq 0.3$ in the case of $|\operatorname{Tr}|=3$. By an extensive study over De and $\mathrm{Tr}$, we found that the condition that good mixing is achieved is $\mathrm{De} \leq 0.1|\mathrm{Tr}|$. If De increases, the area of good mixing reduces as shown by the LIF results in Figures 5(b) and 6(b). The secondary flow pattern approaches that for $\operatorname{Tr}=0$ because the rotation effect of the channel becomes relatively weak as shown by the PIV results of the figures.

Time evolution of the fluctuation of the absolute value of secondary flow velocity (deviation from the mean absolute value of secondary flow velocity) and its Fast Fourier Transform (FFT) at $(x, y)=(-0.64,0.89)(x$ is the horizontal axis and $y$ the vertical axis in the Figures 5 and 6) are shown in Figure 7. Continuous spectrum with a wide range of frequencies, which is characteristic to chaotic motion, is seen in the figure. It was confirmed that the secondary flow becomes chaotic when a good mixing is achieved in the present device.

Mean axial velocity distribution of $\mathrm{De}=0.1|\operatorname{Tr}|$ is shown in Figures 8 and 9. A vertical axis shows mean axial velocity $w$ and the horizontal axis shows $x$ (horizontal axis of Figures 5 and 6). And $y$ is the same as the vertical axis of Figures 5 and $\mathbf{6}$.

Figure 8 is the case for $\operatorname{Tr}=3$ and $\mathrm{De}=0.3$. Compared with Figures 8(b) and (c), the velocity of Figure $\mathbf{8 ( a )}$ is large under the influence of the rotor wall. Also in Figures 8(b) and (c), velocity is increasing in the place near the rotor wall $(x=1.5 \mathrm{~mm})$. Moreover, in Figure $\mathbf{8}(\mathbf{c})$, the reversal of the mean axial flow has been generated from $x=-1.5 \mathrm{~mm}$ (near a casing wall) to $0.5 \mathrm{~mm}$ (near a channel centre). And also in Figure 8(b), the reversal of the mean axial flow has been generated from $x$ $=-1.5 \mathrm{~mm}$ to $0 \mathrm{~mm}$. It is thought that the cause of the occurrence of reversal of the mean axial flow is due to the adverse pressure gradient created by the increase of the exit side pressure under the effect of the rotation of rotor. Figure 9 shows the case for $\mathrm{Tr}=-3$ and $\mathrm{De}=0.3$. In Figure 9, the reversal of the mean axial flow has been generated by the rotation of rotor near the rotor wall. As for Figures 9(b) and (c), because the pressure of the entrance side increased by the rotation of rotor, velocity is large by the favourable pressure gradient. Thus, it is found that when good mixing is realized, a secondary flow becomes chaotic due to destabilization of the unstable reversed mean axial flow.

\subsection{Mixer II and Mixer III}

In this section, we compare the influence of the mixing by the difference of the aspect ratio. The results of LIF and PIV at $\mathrm{De}=0.1|\operatorname{Tr}|$ of Mixer II and Mixer III are 


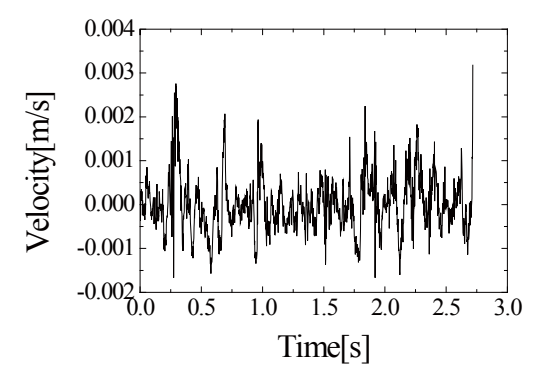

(i) Time evolution of the fluctuation velocity

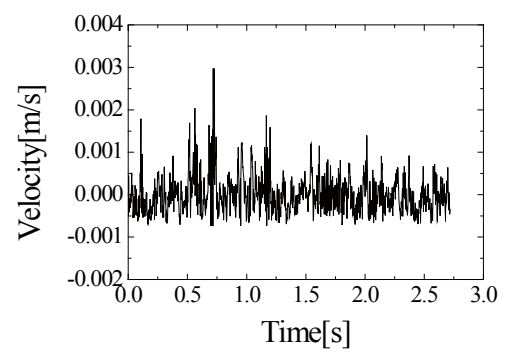

(i) Time evolution of the fluctuation velocity

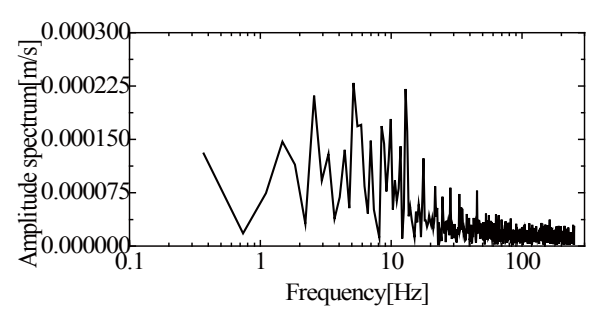

(ii) FFT

(a)

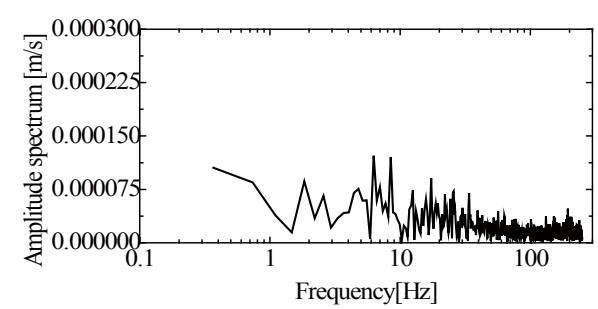

(ii) FFT

(b)

Figure 7. Time evolution of the fluctuation velocity and FFT of Figures 5 and 6 . (a) $\mathrm{Tr}=3$ and $\mathrm{De}=0.3$; (b) $\mathrm{Tr}=-3$ and $\mathrm{De}=$ 0.3.

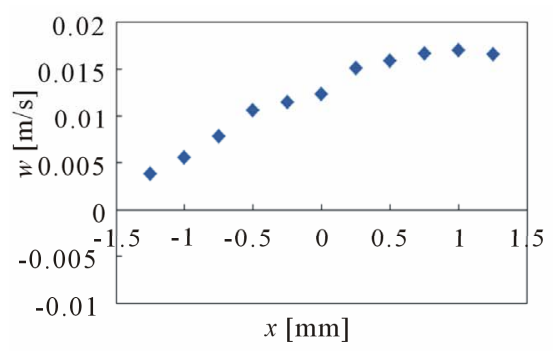

(a)

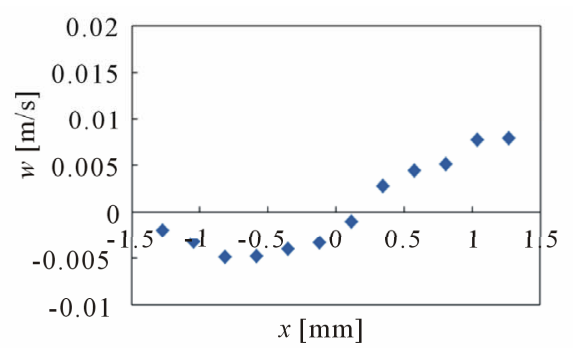

(b)

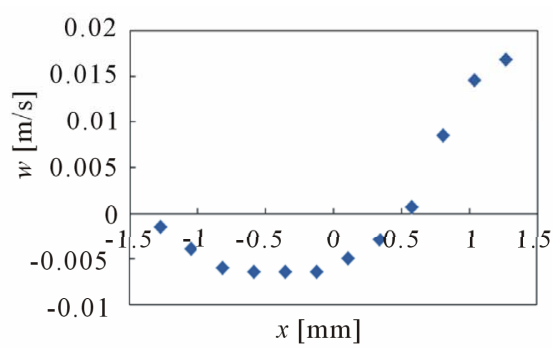

(c)

Figure 8. Axial velocity distribution at $\mathrm{Tr}=3$ and $\mathrm{De}=0.3$. (a) $y=1.0 \mathrm{~mm}$; (b) $y=0.0 \mathrm{~mm}$; (c) $y=-1.0 \mathrm{~mm}$.

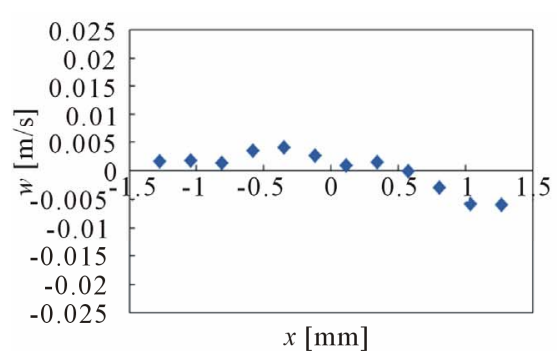

(a)

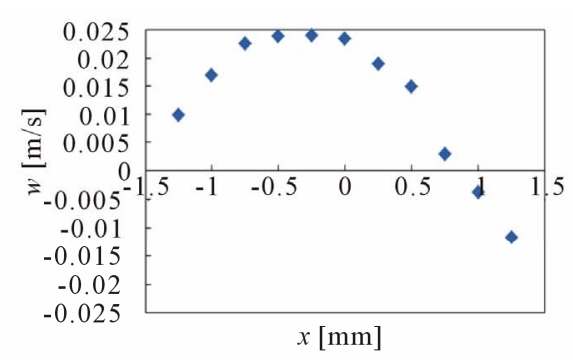

(b)

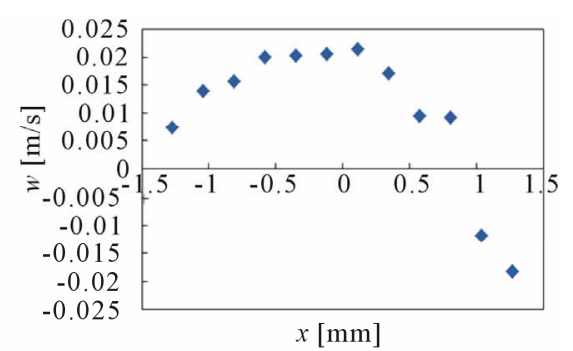

(c)

Figure 9. Axial velocity distribution at $\mathrm{Tr}=-3$ and $\mathrm{De}=0.3$. (a) $y=1.0 \mathrm{~mm}$; (b) $y=0.0 \mathrm{~mm}$; (c) $y=-1.0 \mathrm{~mm}$.

shown in Figures 10 and 11.

From Figures 5 and 10, comparison of the LIF results of Mixer I and Mixer II, Mixer II does not promote mixing like Mixer I. Moreover, when the results of PIV are seen, Mixer II does not have circulating flow like Mixer I. And also, there is no secondary flow (flow to a curved channel inner side) from the casing wall (left) to the rotor wall (right) which generates under the influence of the reversal of the mean axial flow.

Comparison of the LIF results of Mixer I and Mixer III in Figures 5 and 11 shows that mixing is more promoted in the Mixer III than Mixer I, and that the structure of the 


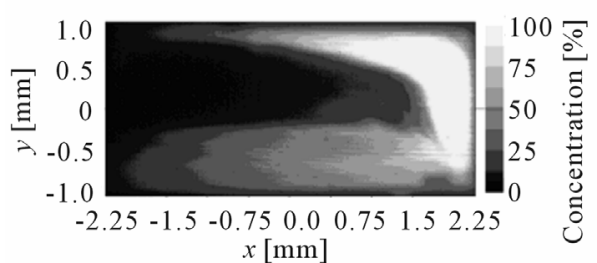

(i) LIF

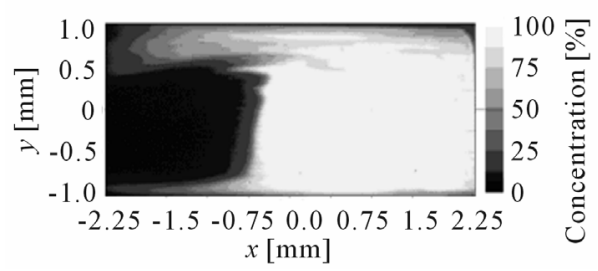

(i) LIF

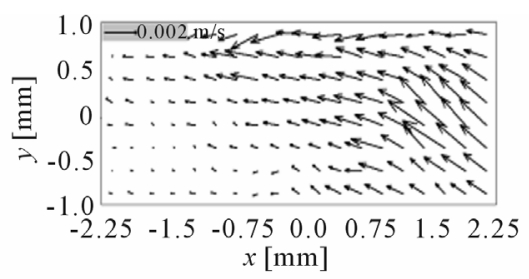

(ii) PIV

(a)

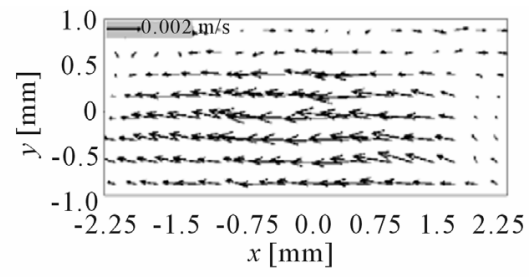

(ii) PIV

(b)

Figure 10. Secondary flow pattern of Mixer II. (a) $\operatorname{Tr}=3$ and $\mathrm{De}=0.3$; (b) $\mathrm{Tr}=-3$ and $\mathrm{De}=0.3$.

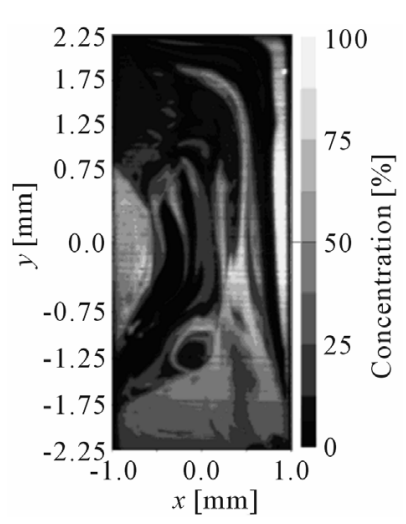

(i) LIF

(a)

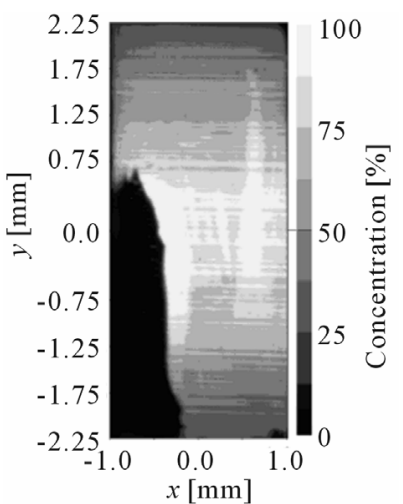

(i) LIF

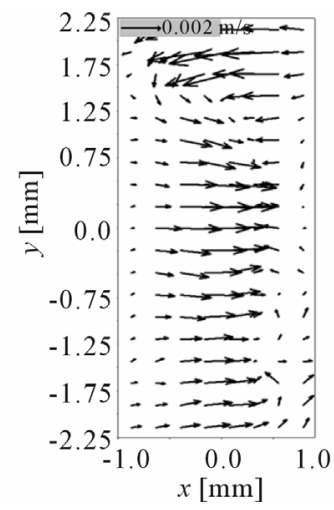

(ii) PIV

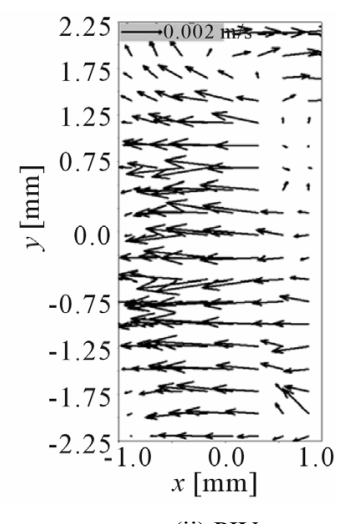

(ii) PIV

(b)

Figure 11. Secondary flow pattern of Mixer III. (a) $\mathbf{T r}=\mathbf{3}$ and $\mathrm{De}=0.3$; (b) $\mathrm{Tr}=-3$ and $\mathrm{De}=0.3$.

secondary flow of Mixer III is more complicated. From the PIV results, it is found that the circulating flow seen in Mixer I is also seen in Mixer III, and that the flow from the casing wall (left) to the rotor wall (right), which is generated under the influence of the reversal of the mean axial flow, exists in large area. It is expected that the promotion of mixing in Mixer III is caused under the strong influence of reversal of the mean axial flow.

\section{Conclusions}

In the present paper, we made the micromixer with a rotor and a curved channel which can mix low Reynolds number flows efficiently, and obtained following results experimentally:

In case of $\gamma=1$ (Mixer I) and $\gamma=2.25$ (Mixer III), the rotation effect of the channel becomes conspicuous for De $\leq 0.1|\operatorname{Tr}|$, and good mixing effect is achieved for those cases. This tendency appears more strongly if $\gamma$ is large and $\operatorname{Tr}>0$.

It is concluded that in case of good mixing, a secondary flow becomes chaotic due to destabilization of the unstable reversed mean axial flow.

\section{Acknowledgements}

The authors would like to express their cordial thanks to Naoyuki Yasuda and Koichiro Tabara for their help in the experiments.

\section{REFERENCES}

[1] A. D. Stroock, S. K. W. Dertinger, A. Ajdari, I. Mezic, H. A. Stone and G. M. Whitesides, "Chaotic Mixer for Microchannels," Science, Vol. 295, No. 5555, 2002, pp. 647 651. doi:10.1126/science. 1066238

[2] D. S. Kim, I. H. Lee, T. H. Kwon and D. W. Cho, "A 
Novel Chaotic Micromixer: Barrier Embedded Kenics Micromixer," Proceedings of 7th International Conference on Miniaturized Chemical and Biochemical Analysis Systems, Squaw Valley, 5-9 October 2003, pp. 73-76.

[3] H. Sato, S. Ito, K. Tajima, N. Orimoto and S. Shoji, "PDMS Microchannels with Slanted Grooves Embedded in Three Walls to Realize Efficient Spiral Flow," Sensors and Actuators A: Physical, Vol. 119, No. 2, 2005, pp. 365-371. doi:10.1016/j.sna.2004.08.033

[4] X. Z. Niu and Y.-K. Lee, "Efficient Spatial-Temporal Chaotic Mixing in Microchannels," Journal of Micromechanics and Microengineering, Vol. 13, No. 3, 2003, pp. 454-462. doi:10.1088/0960-1317/13/3/316

[5] P. Tabeling, M. Chabert, A. Dodge, C. Jullien and F.
Okkels, "Chaotic Mixing in Cross-Channel Micromixers," Philosophical Transactions of the Royal Society A, Vol. 362, No. 1818, 2004, pp. 987-1000. doi:10.1098/rsta.2003.1358

[6] The Japan Society of Mechanical Engineers, Ed., "JSME Data Book: Hydraulic Losses in Pipes and Ducts," The Japan Society of Mechanical Engineers, Tokyo, 1971, pp. 68-72.

[7] K. Yamamoto, X. Y. Wu, K. Nozaki and Y. Hayamizu, "Visualization of Taylor-Dean Flow in a Curved Duct of Square Cross-Section," Fluid Dynamics Research, Vol. 38, No. 1, 2006, pp. 1-18. doi:10.1016/j.fluiddyn.2005.09.002 